\title{
IDENTIFICATION OF THE IB EMISSION BAND IN Al-DOPED ZnS CRYSTALS
}

\author{
N.Q. Liem, V.X. Quang and D.X. Thani \\ Institute of Material Science, Nghia do-Tu liem, Hanoi, Vietnam
}

(Received February 19, 1996; in final form May 21, 1996)

\begin{abstract}
Time-resolved luminescence spectroscopy and thermal lensing techniques are applied to the study of deep centre photoluminescence and absorption in Al-doped $\mathrm{ZnS}$ crystals, which are as-grown or heat treated in different $\mathrm{Zn}$ rich or $\mathrm{S}$ rich atmospheres. A blue emission band peaking at $2.89 \mathrm{eV}$ has been identified as IB band in all the mentioned crystals. Parameters of the IB band at $300 \mathrm{~K}, 77 \mathrm{~K}$ and $10 \mathrm{~K}$ such as peak energy, half width of spectrum, radiative lifetime have been determined. The IB band exhibits some special properties such as a shift neither with increasing delay time nor with excitation intensity nor with sample temperatures as well as a superlinearly excitation intensity dependence of the luminescence. An energy diagram and electronic transitions in the IB centre are presented to explain the experimental results.
\end{abstract}

PACS numbers: 78.55.-m, 78.55.Et

\section{Introduction}

Zinc sulphide is one of the typical members of II-VI compounds. Its direct and wide band gap promises a good application in the optoelectronic and luminophor field, especially operating in the short wavelength visible region [1-3]. From the application point of view, $\mathrm{ZnS}$ should be doped with many different suitable kinds of activators or/and coactivators. In general these dopants and the vacancies created during doping process can become the efficient luminescent centres, which are single or complex together. Aluminium is an important coactivator when codoping with the activators such as $\mathrm{Ag}$ or $\mathrm{Cu}[4,5]$. Moreover, in $\mathrm{ZnS}$ crystal Al can act as a donor and as a component in the aluminium-zinc vacancy $\left(V_{Z_{n}}\right)$ complex, which is responsible for the very well-known emission being the so-called SA band [6-8]. Depending on the impurities and lattice damage states of $\mathrm{ZnS}$ crystals there exist some luminescence bands at high energy side of the usual SA. For example, the B-Cu band peaking at $450 \mathrm{~nm}$ of $\mathrm{ZnS}: \mathrm{Cu}[9]$ and of $\mathrm{ZnS}: \mathrm{Cu}, \mathrm{Mn}[10]$; the $\mathrm{HB}$ band at $3.05 \mathrm{eV}$ of cubic $\mathrm{ZnS}: \mathrm{Cl}[11]$; an unidentified 
emission band near $2.88 \mathrm{eV}$ observed occasionally on $\mathrm{ZnS}: \mathrm{Al}[12,13]$; the IB band peaking at $2.91 \mathrm{eV}$ of undoped $\mathrm{ZnS}$ [14] and at about $2.87 \mathrm{eV}$ of the ion implanted $\mathrm{ZnS}: \mathrm{Al}$ crystals [6]; the blue band peaking at $425 \mathrm{~nm}$ for undoped $\mathrm{ZnS}$ [15] and $430 \mathrm{~nm}$ for Cu-, Ag-, $\mathrm{Mn}$-doped $\mathrm{ZnS}[8,16]$. We will discuss these emissions more clearly later. Among the above bands there is a band family originated from the centres associated with the same $\mathrm{Zn}$-vacancy type. $\mathrm{V}_{\mathrm{Zn}}$ can be created by many different manners, e.g. by ion implantation or by heat treatment $[6,16]$. Treatments of crystals in different ambient conditions will change the vacancy concentrations. In such a case it is possible to study more separately the emission band originated from the centre, which is associated with the $\mathrm{V}_{\mathrm{Z}_{n}}$ lattice damage. We have already reported the IB emission of undoped ZnS [14], but only some preliminary results of $\mathrm{Al}$-doped $\mathrm{ZnS}$, and confirmed that $\mathrm{Zn}$-vacancy played a very important role in the configuration of the IB centre [6].

The purpose of this paper is to determine the detailed parameters as well as properties of the IB band in Al-doped $\mathrm{ZnS}$ crystals, which were as-grown and heat treated. We are also trying to identify it with some emission bands observed occasionally in pure and doped ZnS crystals on the basis of the nature of the transitions. An energy diagram and electronic transitions in the IB centre are presented to explain the experimental results.

\section{Experimental}

The samples for investigation were cut from the bulk Al-doped $\mathrm{ZnS}$ single crystal, which was grown by melting method. The aluminium concentration was assumed to be of $10^{17} \mathrm{~cm}^{-3}$ from the starting material ratios. In order to control the lattice damage concentration, some treatment technologies were applied to the samples as follows:

- Heat treatment in molten zinc for 74 hours at $900^{\circ} \mathrm{C}$ to purify and to perfect crystal (i.e. the solvent extraction purification method [17], herein we call it the sample type I);

- Annealing at $400^{\circ} \mathrm{C}$ under saturated $\mathrm{Zn}$ vapour to reduce $\mathrm{Zn}$-vacancy amount (we call it the sample type II);

- Annealing at $400^{\circ} \mathrm{C}$ under $\mathrm{H}_{2}$ stream to increase $\mathrm{Zn}$-vacancy concentration (sample type III).

The annealing and heat treatment experiments have been described in our previous paper [6]. All the above samples were investigated by photoluminescence (PL) techniques included both stationary (herein we call it dc-PL) and pulsed excitations. In the case of dc-PL the excitation rate is estimated to be about $10 \mathrm{~mW} / \mathrm{cm}^{2}$, while that in the case of pulsed excitation changed in four orders of magnitude from $200 \mathrm{~kW} / \mathrm{cm}^{2}$ down to $20 \mathrm{~W} / \mathrm{cm}^{2}$. Some different features of the time-resolved technique would be profited to study the nature of the emission bands. These PL techniques have been clearly described in Ref. [14]. The sample temperatures could be varied from $300 \mathrm{~K}$ down to $10 \mathrm{~K}$ by using a helium cryostat of Chemical Cryogenics Incorporation.

In order to determine the characteristic absorption of the deep centres within the band gap, a thermal lensing technique was performed. This is considered as one 
of the supersensitive methods to study the weak nonradiative transitions, which are correspondent to the absorption coefficient as small as $10^{-5} \mathrm{~cm}^{-1}$. Due to supersensitivity the thermal lensing technique has been efficiently used to study two-photon absorption in molecules $[18,19]$ and firstly applied to the investigation of semiconductor [20]. Briefly, the principle of the method can be demonstrated as follows: when material absorbs energy from a laser beam at least a fraction of this optical excitation energy will be deformed into heat. If the laser beam has Gaussian intensity distribution the temperature field of medium should change with the same Gaussian shape. Each material has a specific coefficient $\mathrm{d} n / \mathrm{d} T$, which describes the variation of refractive index with temperature. This leads to a formation of a thermal microlens inside the medium when it is heated by laser beam. Now, if a second laser beam passes transparently through such area it will be diverged or focused depending on the value of $\mathrm{d} n / \mathrm{d} T$ being negative or positive, respectively. The first laser is the so-called heating beam, which is normally a pulsed dye laser. The second one is the so-called probe beam being generally IIe-Ne laser. The variation of intensity of probe beam detected in the central spot far away from the sample will be proportional to the absorption of material. Therefore, an absorption-like spectrum against the excitation energy can be registered. In our thermal lensing experiment energy of the heating beam varied from 2.5 to $3.0 \mathrm{eV}$, which covered enough the absorption range of the IB band. Due to sublimation causing roughness of a sample surface, which affects strongly the signal, some precautions must be taken in order to present the attenuation of the excitation intensity by the calibrated neutral-density filters and lenses. The theoretical treatment of the experimental results, i.e. calculations, fitting procedures, etc. have been described elsewhere [14].

\section{Results and discussions}

In all of the undoped and doped $\mathrm{ZnS}$ crystals at $300 \mathrm{~K}$ and $77 \mathrm{~K}$ under stationary excitation by $365 \mathrm{~nm}$ light of mercury high pressure lamp we obtained only the SA emission band, although its intensity varied from sample to sample. The SA band, in our case peaking at about $2.65 \mathrm{eV}$ at $77 \mathrm{~K}$, has the spectroscopic parameters and characteristic properties such as peak energy, spectral half width as well as a shift positively following the sample temperature, etc. the same as those in the literature $[7,8]$. When the samples are excited by $333 \mathrm{~nm}$ the IB band appears obviously at liquid nitrogen or lower temperature. Figure 1 shows dc-PL spectrum at $77 \mathrm{~K}$ of as-grown $\mathrm{Al}$-doped $\mathrm{ZnS}$ crystal excited by $333 \mathrm{~nm}$ line of mercury lamp. Using the computation techniques mentioned above and the determined data of the SA band we could do a curve fitting to receive fairly the IB emission band peaking at around $2.89 \mathrm{eV}$. The IB emissions in all the samples are identified on the basis of the spectrum characteristics such as peak energy, spectral half width as well as specific PL excitation (PLE) spectra (Fig. 1B). It can be seen while comparing the intensities of the IB $\left(I_{\mathrm{IB}}\right)$ and SA $\left(I_{\mathrm{SA}}\right)$ bands of this sample to those of undoped $\mathrm{ZnS}[14]$ that $I_{\mathrm{IB}} / I_{\mathrm{SA}}$ ratio is smaller in this case. This is quite understandable because in the present study the samples are doped with $\mathrm{Al}$, which enhances the SA emission. The above intensity ratio of the type I sample is the smallest, while that of type III or as-grown samples is the largest; it 


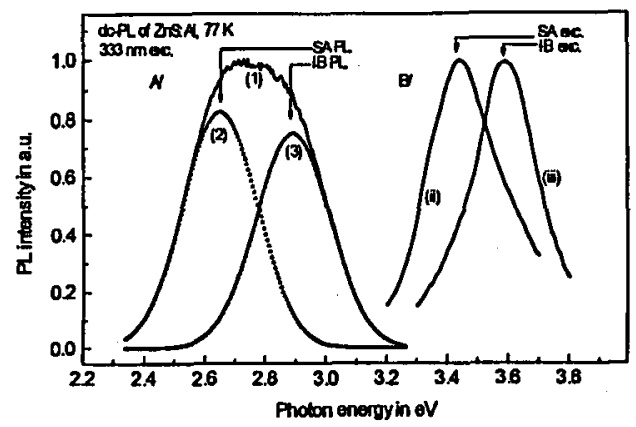

Fig. 1. (A) dc-PL of as-grown Al-doped $\mathrm{ZnS}$ crystal at $77 \mathrm{~K}$ excited by $333 \mathrm{~nm}(1)$, Gaussian fits for the SA (2) and IB (3) components. (B) dc-PLE spectra of the SA (ii) and IB (iii) corresponding to the above PL bands.

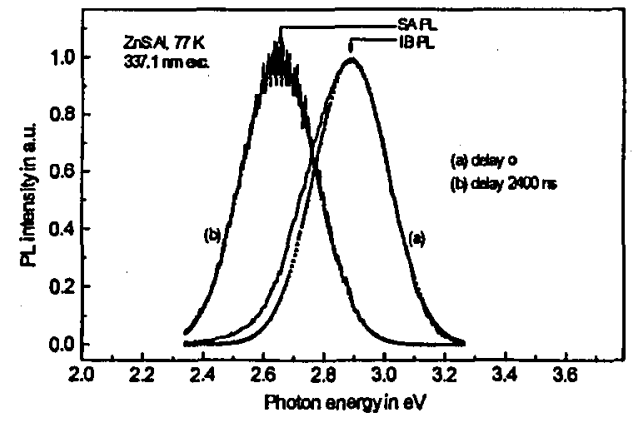

Fig. 2. Time-resolved PL of as-grown Al-doped $\mathrm{ZnS}$ crystal at $77 \mathrm{~K}$ excited by $200 \mathrm{~kW} / \mathrm{cm}^{2}$ pulsed nitrogen laser: (a) PL was recorded just after excitation (solid line) and Gaussian fit for the IB (symbolled line); (b) PL recorded delaying $2.4 \mu$ s after the excitation (solid line) and Gaussian fit for the SA (symbolled line).

is intermediate for the sample type II. To explain this effect it must be remembered that $\mathrm{Zn}$-vacancy played a very important role in the IB centre configuration [6] and its concentration varied in the samples undergone heat treatments. In our opinion, in as-grown crystals a lot of thermally induced vacancies always exist. This is the reason why the IB emission band appears strongly in these crystals.

From PLE spectrum it can be seen that the nitrogen laser is very suitable to excite the IB luminescence. The PL lifetime measurements of all samples at 300 and $77 \mathrm{~K}$ were done with a pulsed nitrogen laser excitation. The respectively obtained values of the IB luminescence are 110 and $400 \mathrm{~ns}$, which are much smaller than those of the SA emission, namely 2.4 and $4 \mu \mathrm{s}$. From this difference of lifetimes it is convenient to study distinguishably PL of each the IB or SA bands by the time-resolved PL technique. Figure 2 shows the time-resolved PL spectra of as-grown Al-doped $\mathrm{ZnS}$ crystal at $77 \mathrm{~K}$ excited by the pulsed nitrogen laser. It can be clearly seen that PL recorded just after excitation is almost the IB, while that recorded delaying $2.4 \mu$ s after the excitation is the SA band only. The curve 
fittings for the IB and SA bands are also presented showing very good agreement between the experimental data and Gaussian distribution. In the present paper the IB band is separately deducted from the common spectrum by the time-resolved technique so that its proper parameters can be determined accurately more than those of the primary paper [6].

We try to profit some different features of the time-resolved technique to research the nature of the IB emission in Al-doped $\mathrm{ZnS}$ crystals. The pulsed nitrogen laser was used as the excitation source in two different series of experiments performed as follows:

(i) Keeping the same specified delay time after excitation (at 0, 30, 50, $100 \mathrm{~ns}$ long to $2400 \mathrm{~ns}$ elapsed) to measure the spectrum shift following excitation rates from $200 \mathrm{~kW} / \mathrm{cm}^{2}$ down to $20 \mathrm{~W} / \mathrm{cm}^{2}$. This is the so-called $J$-shift. The results showed that the IB emission peak moved very slightly to the lower energy side corresponding to the lower excitation rate, while the common half width was expanded. The curve fits for the IB and SA bands were carried out in order to separate these components. The final results showed that only the SA band shifted as a consequence of donor-acceptor (D-A) pair transitions, but the IB band did not move at all. The slight $J$-shift shown above is caused by a superposition of the SA and IB bands. From the results obtained in this experimental series it can be also drawn the luminescence intensity dependence on the excitation intensity. The emission intensity of the SA band varied almost linearly on the excitation rate change and occupied a small range in the normalized spectra only (see Fig. 2a typically). The IB emission, in other way, depends superlinearly on the excitation rate. Figure 3 shows curves based on the equation $I \propto L^{k}$, where $I$ is the IB

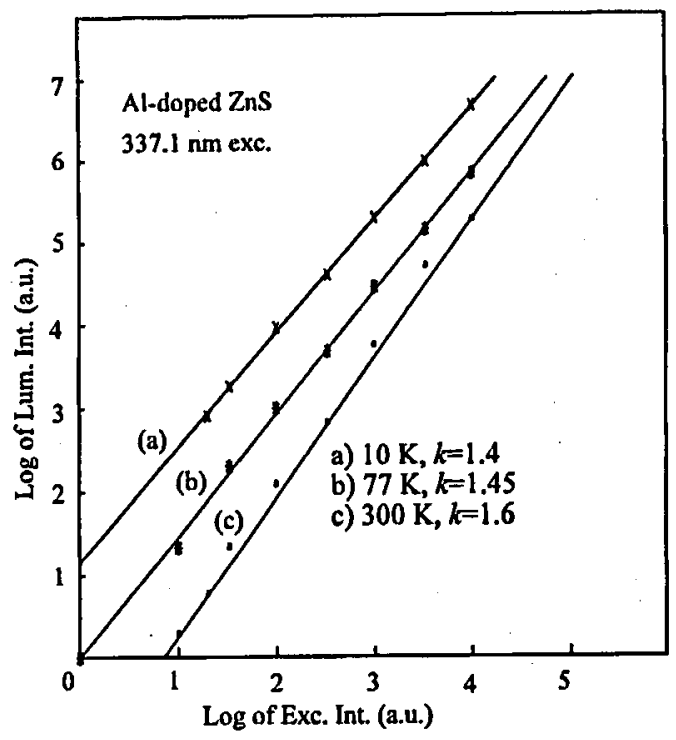

Fig. 3. The IB PL dependence on the excitation rate of as-grown Al-doped ZnS crystal at 300,77 and $10 \mathrm{~K}$. PL was recorded just after excitation pulse. 
emission intensity, $L$ is the excitation intensity and $k$ is the superlinear coefficient. The values of $k=1.6,1.45$ and 1.4 were received for $\mathrm{ZnS}: \mathrm{Al}$ crystals at 300 , 77 , and $10 \mathrm{~K}$, respectively. These $k$ values are slightly smaller than those of the undoped samples [14], probably because of the fact that the SA centre operated as an energy dissipated channel. It can be seen that intensity quenching of the IB emission is rather strong and becomes stronger at higher temperature. On the basis of such facts as the larger $k$ value and shorter lifetime of the IB emission at higher temperature it is possible to understand why in most dc-PL spectra the IB band was not clearly visible beside the SA band.

(ii) Keeping the excitation at a fixed rate to measure the spectrum shift following a delay time after excitation. This is the so-called $t$-shift. The time-resolved spectra were recorded at different delay times $0,30,50,100,150,300,600,1000$, 1500 and $2400 \mathrm{~ns}$ after excitation and at the sample temperatures of 300,77 and $10 \mathrm{~K}$. The results of this experimental series were similar to those of the $J$-shift ones, e.g. a small movement of the common peak was caused by the superposition of the SA on the decayed IB emission. The lower excitation rate, the larger $t$-shift is consequently a share of the band superposition and the superlinear excitation intensity dependence of the IB luminescence. However, all of these shifts are very small and result in an expansion of the common spectrum. For much longer delays, only the SA band could be observed and was shifted following delay times as a consequence of D-A transitions. The curve fitting for the IB and SA bands showed that there is no $t$-shift of the IB band.

In addition to the shift behaviour of the IB emission, we measured the band shift following temperatures (it is the so-called $T$-shift) from $300 \mathrm{~K}$ down to $10 \mathrm{~K}$. The IB emission was recorded at a fixed moment just after excitation. There is no $T$-shift of the IB band, either.

The parameters and properties of the IB band in $\mathrm{ZnS}: \mathrm{Al}$ are collected in Table. $E_{\mathrm{p}}$ is the peak energy and $W$ is the half width of the IB band; $\tau$ is the lifetime of the IB transition. The $J-, t$-, and $T$-shifts of the luminescence spectra have a usual meaning as explained above and in other publications.

\section{TABLE}

Spectroscopy parameters and characteristic properties of the IB emission band of Al-doped ZnS crystal at different temperatures.

\begin{tabular}{l|c|c|c|c|c}
\hline \hline & $300 \mathrm{~K}$ & $200 \mathrm{~K}$ & $130 \mathrm{~K}$ & $77 \mathrm{~K}$ & $10 \mathrm{~K}$ \\
\hline$E_{\mathbf{p}}[\mathrm{eV}]$ & 2.89 & 2.89 & 2.89 & 2.89 & 2.89 \\
$W[\mathrm{meV}]$ & 406 & - & - & 300 & 270 \\
$\tau[\mathrm{ns}]$ & 110 & - & - & 400 & - \\
$J$-shift & no & - & - & no & no \\
$t$-shift & no & - & - & no & no \\
$T$-shift & no & - & - & no & no \\
$k$ & 1.6 & - & - & 1.45 & 1.4
\end{tabular}


We now discuss the transitions responsible for the IB emission in more detail. Due to the Coulomb term $e^{2} / \varepsilon r$ (herein $\varepsilon$ is the static dielectric constant and $r$ is the $\mathrm{D}-\mathrm{A}$ pair separation) added to the radiative energy of $\mathrm{D}-\mathrm{A}$ pair recombination the signs for recognition, if the transition belongs to D-A pairs, are the $J$-shift and $t$-shift behaviours of the spectra. The concentration of the D-A pairs changes the average pair distance $r$ so that it contributes to the shift of the band, too [15]. From all the obtained results shown no peak shift with the mentioned parameters we can confirm that the nature of the IB transition is unlikely D-A pair recombination. It must be kept in mind here that our samples were intentionally doped with aluminium, which is potentially to result in a donor state in II-VI compounds and furthermore giving rise to D-A recombination. However, this did not happen to the IB emission, giving the meaning that $\mathrm{Al}$ was probably involved in a complex. It was also stated that $\mathrm{V}_{\mathrm{Zn}_{n}}$ played a very important role in the IB centre configuration [6]. Thus the complex corresponding to the IB centre would consist of $\mathrm{V}_{\mathrm{Zn}}$ and $\mathrm{Al}$ but seems unlikely to be the pure A-centre, i.e. the neutrally charged $\left(\mathrm{V}_{\mathrm{Zn}}-\mathrm{Al}_{\mathrm{Zn}}\right)$ complex as the one described in Ref. [21], due to the following reasons: firstly, if it could take part in the D-A pair as an acceptor, the A-centre would have to lead to the SA emission; secondly, the energy depth $0.1 \mathrm{eV}[22]$ below the conduction band of $\mathrm{Al}$ donor is too small as compared to the difference between peak energies of the SA and IB bands. Therefore, the transition of electron from the conduction band to the A-centre acceptor alone could not result in the IB band. We propose here a model of the deep acceptor responsible for the IB transition to be more complicated than a complex of the singly negatively charged $\mathrm{A}$-center $\left(\mathrm{V}_{\mathrm{Zn}}-\mathrm{Al}_{\mathrm{Zn}}\right)^{-}$and an unidentified part involved in other lattice defects. Concretely the IB centre is symbolized as $\left[\left(\mathrm{V}_{\mathrm{Zn}_{\mathrm{n}}}-\mathrm{Al}_{\mathrm{zn}}\right)^{-}+\ldots\right]$. The singly negatively charged A-center acceptor lies at about $3.4 \mathrm{eV}$ below the conduction band, which corresponds to the characteristic excitation of the SA emission [6]. The peak energy of the IB band in Al-doped $\mathrm{ZnS}$ locates at $2.89 \mathrm{eV}$ being slightly smaller than that in the undoped crystals (i.e. $2.91 \mathrm{eV}$ [14] or $425 \mathrm{~nm}$ [15]). This result is quite reasonable due to the fact that the more complex IB centre can induce the deeper acceptor in the band gap. Some other consequences affected the IB emission by aluminium dopant in ZnS crystal can be observable to be as the slight shorter lifetime and broader spectral half width as compared to those of the undoped one. In order to determine the energy level corresponding to the IB centre the absorption spectra of the Al-doped $\mathrm{ZnS}$ samples were measured. In Fig. 4 the absorption-like spectrum measured by the thermal lensing technique is shown. It is clearly seen that there is only one band peaking at almost the same energy as that of the emission. This is the direct absorption by the IB centre. The lack of other absorption bands in the lower energy side means that there is only the transition of electron from the deep acceptor to the conduction band, but not to the donor levels at all.

Basing on all the spectroscopy characteristics and properties obtained for the IB band, the transitions resulted in the IB emission can be illustrated in Fig. 5. The numbers marked the ordinal transitions, which were clearly explained in Ref. [14]. Briefly, the transitions in Fig. 5 are as follows: 


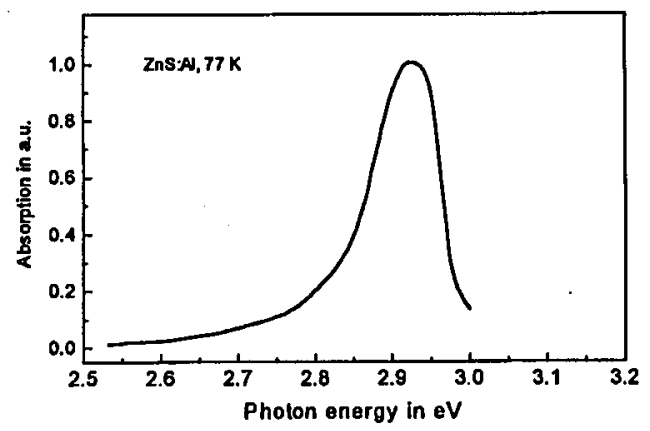

Fig. 4. Absorption-like spectrum of as-grown Al-doped $\mathrm{ZnS}$ at $77 \mathrm{~K}$ measured by the thermal lensing technique.

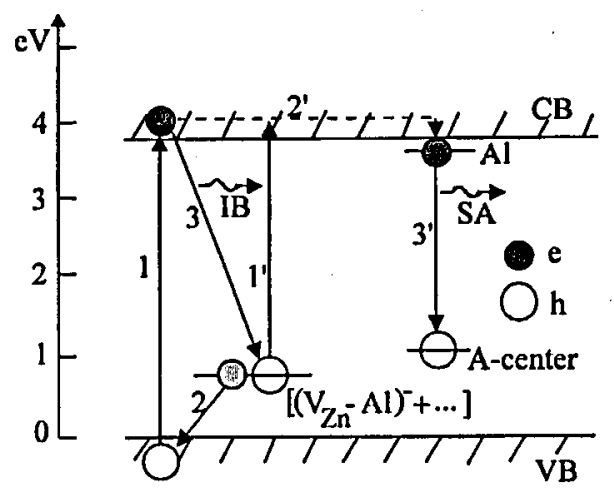

Fig. 5. Transitions responsible for the IB emission of Al-doped ZnS crystal. The detailed explanations are in the text.

(1) Process 1 causes absorption and creates electrons in the conduction band with the same number of holes in the valence band. The direct absorption of the IB centre is marked by number $1^{\prime}$;

(2) The electrons from the $\left[\left(\mathrm{V}_{\mathrm{Zn}_{\mathrm{n}}}-\mathrm{Al}_{\mathrm{Zn}_{\mathrm{n}}}\right)^{-}+\ldots\right]$ complex should fall down to the valence band (marked by number 2). One part of conduction electrons should also relax to a certain donor, which is aluminium in this case (marked $2^{\prime}$ );

(3) Recombination of the electrons and holes from the conduction band and $\left[\left(\mathrm{V}_{\mathrm{Zn}_{\mathrm{n}}}-\mathrm{Al}_{\mathrm{Zn}_{\mathrm{n}}}\right)^{-}+\ldots\right]$ complex, respectively, emits the IB luminescence (marked 3$)$, while this results from Al-donor and A-centre in the SA band (marked $3^{\prime}$ ).

Depending on the concentrations of the donors and acceptors in the crystals, the presented model could explain the superlinear excitation intensity dependence of the IB luminescence. The superlinear dependence on the excitation of the deep centre PL has been also observed in CdS [23]. Some publications have reported a superlinear excitation power dependence of the near band edge PL of CdTe compound $[24,25]$. It can be estimated that the excitonic processes would result in an emission with a quadratic dependence on the excitation rate if this excita- 
tion causes the electron concentration in the conduction band being equal to the concentration of holes in the valence band and there is no other nonradiative relaxation channels [24]. In our case, the IB PL yielded from the transitions between the deep acceptor and two conduction and valence bands so that it is possible to apply the theory proposed for the excitonic processes, of course with some different initial conditions. The existence of some certain relaxation channels (e.g. the SA) leads to the superlinear dependence on the excitation rate of the IB emission with the coefficient $k$ lying between 1 and 2 .

In the past, some authors wrote sketchily about an existence of a luminescence band, which we are trying to identify with the IB band as below. When measuring the decay characteristics of the SA emission in $\mathrm{ZnS}: \mathrm{Cl}$ the fast decay component with a time constant being about $400 \mathrm{~ns}$ at $77 \mathrm{~K}$ was shown in Ref. [26]. During the study by time-resolved optically detected magnetic resonance (ODMR) in $\mathrm{ZnS}: \mathrm{Al}, \mathrm{I}$ the small contribution at $430 \mathrm{~nm}$ at a short delay time showed no shift with increasing delay time and would be attributed to D-A transition of Cu-blue centre relaxed after annealing [8]. Perhaps this band was unreasonably attributed to the $\mathrm{Cu}$-blue centre, because the $\mathrm{Cu}$-blue emission has been well identified peaking at $450 \mathrm{~nm}[9,10]$. The unidentified emission band near $2.88 \mathrm{eV}$ observed occasionally on $\mathrm{ZnS}: \mathrm{Al}$ without peak position shift in the time-resolved experiments was shown $[12,13]$. In our opinion, in each of the above papers $[6,8,12,13,26]$ the authors partly studied the characteristics of the same blue emission band, which we identified as the IB band in the doped $\mathrm{ZnS}$ crystals. In Ref. [14] the IB luminescence band has been identified for the undoped $\mathrm{ZnS}$ samples. It is possible to assign the name IB for the blue emission band peaking at $2.89 \mathrm{eV}(430 \mathrm{~nm}[8,16], 2.88 \mathrm{eV}[12,13])$ of the doped and at $2.91 \mathrm{eV}(425 \mathrm{~nm}$ [15]) of the undoped $\mathrm{ZnS}$ on the basis of the same native $\mathrm{Zn}$-vacancy involved in the centre and the same spectroscopy characteristics.

\section{Conclusions}

The time-resolved spectroscopy and thermal lensing techniques are applied to the study of deep centre photoluminescence and absorption in Al-doped ZnS crystals. On the higher energy side of the usual SA emission there exists the blue emission band named the IB. This band of the Al-doped $\mathrm{ZnS}$ crystals has been studied relatively in detail. Basing on the spectroscopy characteristics such as peak energy, radiative life, as well as spectral half width and properties such as no $J$-shift, no $t$-shift, etc., of the IB emission we have tried to identify it with some emission bands observed occasionally or studied sketchily in the pure and doped $\mathrm{ZnS}$ crystals. The experimental results support the model conduction band-acceptor transitions of the IB luminescence. This deep acceptor is proposed to be the complex of the singly negatively charged A-center and the unidentified part, which is involved in other lattice defects. The energy diagram and electronic transitions in the IB centre are presented. In our opinion, the IB centre exists and has been systematized in the undoped or doped ZnS crystals; it has native nature like the SA centre case. Investigations of the electronic and atomic structures of the IB centre can be efficiently performed by the polarised PL, EPR or ODMR experiments. 


\section{Acknowledgments}

The authors wish to thank Mr. N.V. Quang and Mr. N.V. Manh for technical service. One of the authors (NQL) is greatly indebted to Profs. S. Califano and P.R. Salvi for working facilities and to TWAS for a grant.

\section{References}

[1] R.A. Reynolds, J. Vac. Sci. Technol. A 7, 269 (1989).

[2] R. Gunshor, A. Nurmikko, M. Kobayashi, Phys. World March 1992, p. 46.

[3] A.H. Kitai; Solid State Luminescence, 1st ed., Chapman and Hall, London 1993, p. $133,365$.

[4] R. Raue, M. Shiiki, H. Matsukiyo, H. Toyama, H. Yamamoto, J. Appl. Phys. 75, 481 (1994).

[5] S. Kuboniwa, H. Kawai, T. Hoshina, Jpn. J. Appl. Phys. 19, 1647 (1980).

[6] V.X. Quang, T.K. Anh, N.Q. Liem, K. Mass, B. Selle, Phys. Status Solidi A 79, K181 (1983).

[7] A. Abdel-Kader, II.I. Farag, J. Mater. Sci. 23, 2382 (1988).

[8] P. Dawson, B.C. Cavenett, J. Lumin. 18/19, 853 (1979).

[9] P. Peka, H.-J. Schulz, Physica B 193, 57 (1994).

[10] E. Chimczak, M. Bertrandt-Zytkowiak, R. Czaika, W.S. Gordon, Acta Phys. Pol. A 63, 595 (1983).

[11] S. Oda, H. Kukimoto, J. Lumin. 18/19, 829 (1979).

[12] K. Era, S. Shionoya, Y. Washizawa, J. Phys. Chem. Solids 29, 1827 (1968).

[13] K. Urabe, S. Shionoya, J. Phys. Soc. Jpn. 24, 543 (1968).

[14] N.Q. Liem, V.X. Quang, D.X. Thanh, Acta Phys. Pol. A 86, 979 (1994).

[15] J. Zhou, H. Goto, N. Sawaki, I. Akasaki, J. Lumin. 35, 255 (1986).

[16] A.I. Bazhin, E.M. Malinenko, Acta Phys. Pol. A 73, 279 (1988).

[17] M. Aven, H.H. Woodbury, Appl. Phys. Lett. 1, 53 (1962).

[18] D.S. Kliger, Accounts Chem. Res. 13, 129 (1980).

[19] R. Bini, P. Foggi, N.Q. Liem, P.R. Salvi, Chem. Phys. Lett. 151, 236 (1988).

[20] N.Q. Liem, D.X. Thanh, P.R. Salvi, to be published in Comm. Phys. (1996).

[21] J.E. Nicholls, J.J. Davies, B.C. Cavenett, J.R. James, D.J. Dunstan, J. Phys. C, Solid State Phys. 12, 361 (1979).

[22] H. Kukimoto, S. Shionoya, T. Koda, R. Hioki, J. Phys. Chem. Solids 29, 935 (1968).

[23] Y: Shiraki, T. Shimada, K.F. Komatsubara, J. Appl. Phys. 45, 3554 (1974).

[24] D.E. Cooper, J. Shirafuji, P.R. Newmann, J. Cryst. Growth 86, 544 (1988).

[25] T. Schmidt, G. Daniel, K. Lischka, J. Cryst. Growth 117, 748 (1992).

[26] T. Koda, S. Shionoya, Phys. Rev. A 136, 541 (1964). 